\title{
Recursive Algorithms Based on MTB Tree for Estimating Delays in RC Tree Circuits with considering Charge Sharing Effect
}

\author{
Molin Chang, Shuih-Jong Yih and Wu-Shiung Feng \\ Department of Electrical Engineering, R244 \\ National Taiwan University \\ Taipei, Taiwan, R.O.C. China
}

\begin{abstract}
Internal charges will affect the delay behavior of a CMOS gate. A Modified Threaded Binary (MTB) tree and two recursive algorithms, ECS and EER, are proposed to solve this problem. The charge sharing effect, which takes place among the internal nodes, is also considered in the algorithm ECS.
\end{abstract}

\section{Introduction}

The switch-level simulation is a compromised method between circuit-level and logic-level simulations. It has the advantage of fast speed and good adaptability for VLSI circuit. Some switch-level simulators have been implemented, such as MOSSIM, RSIM [1], [2]. BTS (Binary-tree Timing Simulator) is a switch-level timing simulator based on series-parallel circuit structure [3], which is two or three orders faster than SPICE and has more accurate waveform approximation during the

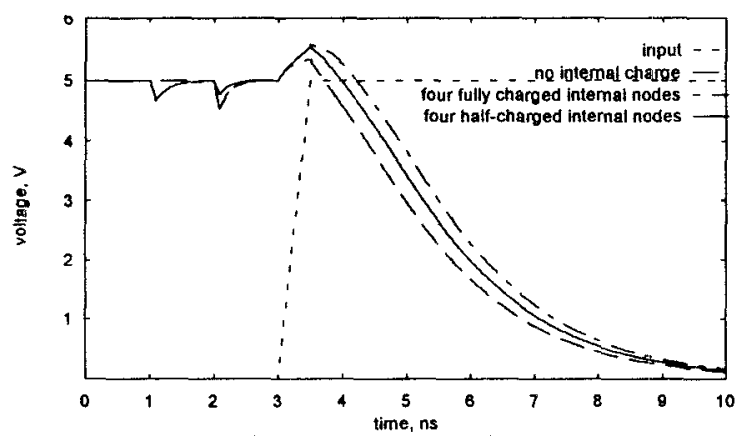

Figure 1: The simulated waveforms of 5 -input NAND gate using SPICE.

transient period.

The internal charges in a CMOS gate will evidently increase the delay. For example, there is a $25 \%$ increment of delay for a five-input NAND gate with four fully charged internal nodes [3]. Therefore, we can not neglect the delay affected by the charges stored in internal nodes. Futhermore, the charge sharing effect acting on the internal nodes will make the calculation work more difficult because the different amount of internal charge leads to different amount of delay. Fig. 1 shows the delay times of 5 -input NAND gate due to the different amount of internal charges. Because of this, we propose two recursive algorithms based on MTB tree to solve this problem.

\section{Series-Parallel Tree}

If a gate is connected by series-parallel way such as fully complementary CMOS, Pseudo-NMOS, Dynamic CMOS and so on, it can be represented as a merged series-parallel tree, also called a $P N$ tree. Fig. 2(b) illustrates the corresponding PN tree of the gate circuit shown in Fig. 2(a) which function is $Z=((a+(b+c)$ $*(d+e)))+f) \#((a *((b * c)+(d * e))) * f)$, where *'s and +'s represent $A N D$ and OR operations, respectively. There are two logical expressions in the equation; the former is the function of $\mathrm{P}$ tree and the latter is of $\mathrm{N}$ tree. The OR nodes in the PN tree are virtual nodes because an OR node only parallels a subcircuit to another and does not create any real node in a gate circuit. For example, if the $n M_{d}$ (the NMOS transistor that accepts the input $d$ ) is removed, the ' $t$ ' is removed simultaneously but no internal node will be removed. Many troublesome problems, such as the evaluation of output state, equivalent RC time constant [3], [4], [5], [6] and internal charge effect, can be solved easily and efficiently by using a series-parallel tree to represent the circuits with the design styles as mentioned above.

\section{MTB Tree}

An MTB tree is also a series-parallel tree, but the threads of a leaf node are always pointed to AND nodes (denoted by $v^{*}$ ). Because OR nodes (denoted by $v^{+}$) are virtual nodes, they must be excluded while establishing the threads of each leaf node. The idea of this new structure is modified from the threaded binary tree [8]; the difference is that the threads of any leaf node are always pointed to AND nodes. In the algorithm ECS that is proposed to solve the problen of charge sharing effect, we need to determine the attribute of each internal node, and probably need to merge two isolated groups or create a new isolated group while visiting the active (turn-on) leaf node in the PN tree. The MTB tree can let us find the adjacent internal nodes of any leaf node directly through its two threads, and thus largely decrease the search time. Fig. 2(b) shows an MTB tree corresponding to the circuit as shown in Fig. 2(a).

\section{Delay Estimation}

BTS is a waveform-based event-driven timing

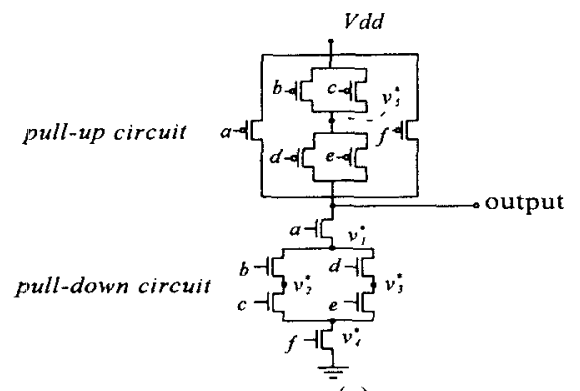

(a)

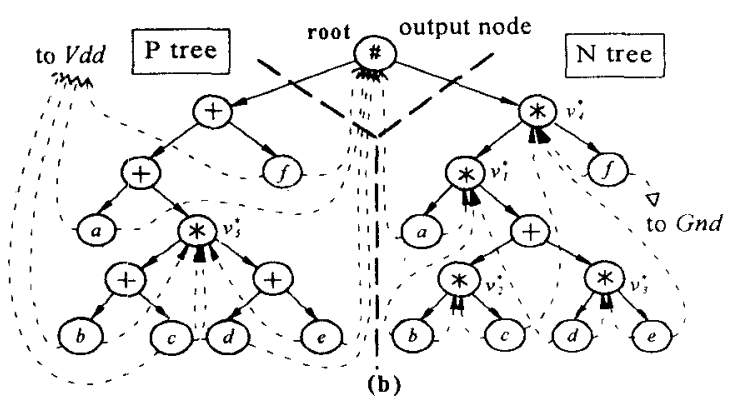

Figure 2: CMOS complex gate. (a) A gate circuit, (b) the equivalent MTB tree

simulator and the primary goal is to obtain an accurate 
waveform [3]. In order to achieve this goal, we adopt the following equations to approximate the waveforms during the transient state [7], and consider the internal charges effect including the charge sharing effect while estimating the delay.

$$
\begin{aligned}
& f=\left\{\begin{array}{ll}
\frac{0.2 t}{T} & t<3 T \\
1-0.4 \exp \left(-\frac{t-3 T}{2 T}\right) & t \geq 3 T
\end{array}\right. \text { for a rising signal } \\
& f=\left\{\begin{array}{ll}
1-\frac{0.2 t}{T} & t<3 T \\
0.4 \exp \left(-\frac{t-3 T}{2 T}\right) & t \geq 3 T
\end{array}\right. \text { for a falling signal }
\end{aligned}
$$

where $\mathrm{T}$ is half of the time spent by signal between $90 \%$ (for a falling signal) or $10 \%$ (for a rising signal) and $50 \%$ of the steady state. If the value of $T$ can be obtained, the transient waveform will then be easily plotted. Hereafter, we only take the falling signal as example, that is, only the $\mathrm{N}$ tree is discussed because of the identical treatment of both.

The delay due to the internal charges can be calculated approximately as

$\Delta t=\frac{Q}{T}=\frac{Q}{V_{s}} 2 R=\frac{\int C d V}{V_{s}} 2 R$

where $\bar{I}$ is the average current, $V_{s}$ is the voltage swing, $R$ is the effective resistance of the conducting path and $Q$ is the charges stored in the internal nodes. More than one internal nodes may be going to charge or discharge in the PN tree, and these nodes must be taken into account when calculating the switching delay. Thus, Eq. 3 is rewritten as $\mathrm{D}_{i}=\sum_{i} 2 R_{i} \frac{Q_{i}}{V_{s}}$

where $R_{i}$ is the effective resistance of internal node $v_{i}^{*}$ with respect to ground, $Q_{i}$ is the charges stored in the internal node $v_{i}^{*}$. For example, if let $V_{a}=V_{c}=V_{d}=V_{f}=5 V, V_{b}=0 V$, and $V_{e}=O V$ to $5 V$ for the circuit as shown in Fig. 2(a), then the internal nodes $v_{1}^{*}$ and $v_{3}^{*}$ are considered because only both nodes have the charges to be discharged. The total delay time caused by the internal charges is $\Delta t=\Delta t_{v^{\ddagger}}+\Delta t_{v_{\xi}^{*}}$, where

$\Delta t_{v_{i}^{*}}=2 R_{v_{i}^{*}} \frac{Q_{v_{i}^{*}}}{V_{s}}=2\left(R_{d}+R_{e}+R_{f}\right) \frac{Q_{v_{i}^{*}}}{V_{s}}$

$\Delta t_{v_{j}^{*}}=2 R_{v_{j}} \frac{Q_{v_{j}^{*}}}{V_{s}}=2\left(R_{e}+R_{f}\right) \frac{Q_{v_{j}}}{V_{s}}$

\section{Charge Sharing Effect}

The states of internal nodes are dynamically changed after a new event occurs, and the output probably changes at this time. No matter what the output state is, we should recalculate the voltage value of each internal node such that the next event can use them as initial values. We observe that the internal nodes in the tree can be classified into three groups, which are (1) $C$ group (nodes in charged state): nodes are connected to output node (the output of this cluster must be in high state), (2) $D$ group (nodes in discharged state): nodes are connected to ground (denoted by $G$ nd) node, and (3) I group (nodes in isolated state) nodes are connected to neither output node nor $G$ nd node. Fig. 3 can illustrate this concept. These groups maybe exist in the tree simultaneously; it depends on the input pattern and the state of output.

To decide the attribute of each node in the tree, we need to traverse the tree by three passes. Each pass should leave a mark on $v^{*}$ that is visited such that any node will be dealt with only once. These marks make the charge sharing problem easier to solve in pass3. Therefore, before presenting the algorithm, a set of symbols to mark the $v^{*}$ will be introduced as follows. $\boldsymbol{H}$ denotes high state, and means that $v^{*} \in C$. $L$ : Output low state $\left(v^{*} \in D\right)$. $\boldsymbol{G}$ : Ground state $\left(v^{*} \in D\right)$. $\boldsymbol{I}_{i}$ : Isolated state $\left(v^{*} \in D\right) . U$ : Undetermined state. All symbols are replaced with an unique integer number in BTS except $\boldsymbol{I}_{i}$, which is changed depending on its group number. We use $M\left(v^{*}\right)$ to represent the mark of $v^{*}$, so $M\left(v^{*}\right) \in\left\{\boldsymbol{H}, \boldsymbol{L}, \boldsymbol{G}, \boldsymbol{I}_{i}, \boldsymbol{U}\right\}$. From experimental results, we assume that the delay of the output changed from HIGH to LOW state is affected by the stored charges and the charge sharing effect in the $\mathrm{N}$ tree [3]. On the contrary, we consider the P tree when output is changed from LOW to HIGH.

Algorithm ECS: (Estimate Charge Sharing effect)

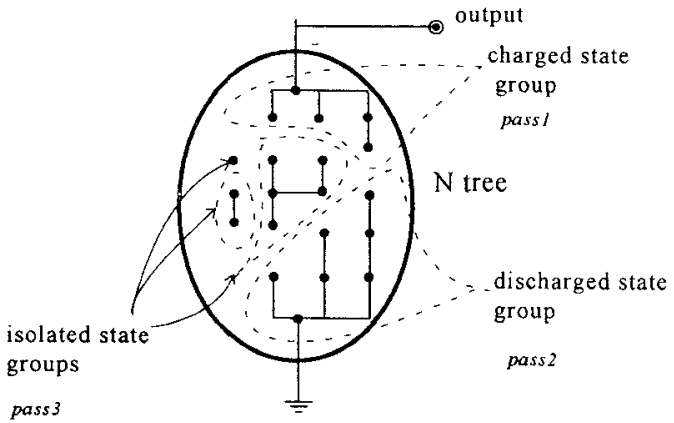

Figure 3: Three states in the $\mathrm{N}$ tree

- Pass 1: Traverse the $\mathrm{N}$ tree recursively by forward inorder visiting (i.e., traverse from left to right). In this pass, all nodes that can reach output node through drain-source channel of the turn-on MOS will be sought out and marked by $\boldsymbol{H}$ or $\boldsymbol{L}$, depending on the state of output. In other words, the $v^{*}$ 's $\in C$ can be almost picked out. This pass will be terminated when the state of any subtree is false.

- Pass 2: Traverse the $N$ tree recursively by backward inorder visiting (i.e., traverse from right to left). The purpose is to seek out the nodes that are connected to Gnd node, i.e., find out the set $\left\{v^{*} \mid v^{*} \in D\right\}$, and also make a mark on them (marked by $\boldsymbol{G}$ ). This pass is also not a complete tree traversal, and the condition of termination is the same as pass 1 .

- Pass 3: Traverse the $\mathrm{N}$ tree recursively by forward preorder visiting. After pass 1 and pass 2 , the most $v^{*}$ 's $\in C$, $D$ can be found out, but some special nodes (called Hidden nodes, which are the nodes that belong to $\boldsymbol{C}$ or $\boldsymbol{D}$ but cannot be sought out in pass 1 or pass 2.) are still not marked. In addition, the isolated nodes $\left(v^{*} \in I\right)$ are also left to be processed by this pass. In this pass, the whole tree is traversed but only the leaf nodes are visited. We can find two adjacent internal nodes of the visited leaf node immediately via two threads of the leaf node. If the leaf node is active, then we can determine the attributes of these two internal nodes by Rule 1 described as follows.

Rule 1: Assume $v_{i}$ is the active leaf node we visited, and then the lchild and the rchild are used to obtain its two adjacent internal nodes, say $v_{i l}^{*}$ and $v_{t r}^{*}$, respectively.

(1) $M\left(v_{i l}^{*}\right)=\boldsymbol{U}$ and $M\left(v_{t r}^{*}\right)=\boldsymbol{H}, \boldsymbol{L}, \boldsymbol{G}$, then $M\left(v_{t i}^{*}\right)=M\left(v_{i r}^{*}\right)$.

(2) $M\left(v_{i l}^{*}\right)=U$ and $M\left(v_{i r}^{*}\right)=I_{i}$, then $M\left(v_{t i}^{*}\right)=M\left(v_{i r}^{*}\right)$.

(3) $M\left(v_{i t}^{*}\right)=U$ and $M\left(v_{i r}^{*}\right)=U$, then $M\left(v_{i i}^{*}\right)=M\left(v_{t r}^{*}\right)=I_{i}+1$, i.e., to create a new isolated group that contains these two nodes. 
(4) $M\left(v_{i l}^{*}\right)=\boldsymbol{I}_{i}$ and $M\left(v_{t r}^{*}\right)=\boldsymbol{I}_{j}, \mathrm{i}<\mathrm{j}$, then $M\left(v_{t r}^{*}\right)=M\left(v_{i l}^{*}\right)$ i.e., the isolated group $I_{j}$ is merged into $I_{i}$.

(5) $M\left(v_{t l}^{*}\right)=\boldsymbol{L}$ and $M\left(v_{t r}^{*}\right)=G$, then $M\left(v_{t r}^{*}\right)=\boldsymbol{L}$.

Note that $v_{i l}^{*}$ and $v_{t r}^{*}$ can be exchanged each other as described in items (1), (2), and (4).

We also use the circuit as shown in Fig. 2(a) as an example to demonstrate this algorithm. Three sequential events are applied to the circuit. Event $1: V a=V c=V d=V f$ $=5 V$, and $V b=V e=0 V$. Pass 1: $M\left(v_{1}^{*}\right)=M\left(v_{3}^{*}\right)=\boldsymbol{H}$. Pass 2: $M\left(v_{2}^{*}\right)=M\left(v_{4}^{*}\right)=G$. Pass 3: no $v^{*}$ needs to be processed. Event 2: $V c=V d=V e=5 V$, and $V a=V b=V f=0 V$. Pass 1: no $v^{*} \in C$. Pass 2: no $v^{*} \in D$. Pass 3: $v_{1}^{*}, v_{2}^{*}, v_{3}^{*}$ and $v_{4}^{*} \in$ $I_{l}$, and charge sharing effect takes place. In theory, there are four 1/2-charged internal nodes in the $\mathrm{N}$ tree. Event 3: $V a$ and $V f$ are changed from $0 \mathrm{~V}$ to $5 \mathrm{~V}$. The output begins to discharge, and its delay should be added by the component resulting from the internal charges stored in nodes $v_{1}^{*}, v_{2}^{*}$, $v_{3}^{*}$ and $v_{4}^{*}$.

\section{Effective Resistance}

In MTB tree, the nodes except leaf nodes are all operators (i.e., *'s and +'s), but only the $v^{*}$ can become the focused node (means that the node attempts to discharge, denoted by $v_{f}^{*}$ ) while calculating the effective resistance ( $R_{e f f}$ ) about it. The calculation of $R_{\text {eff }}$ with respect to $v_{f}^{*}$ is a complicated problem because several paths conducting to Gnd node probably exists in the tree. For example, there are two discharging paths for the node $v_{2}^{*}$ (in Fig. 2(a)), which are $n M_{b}-n M_{d} n M_{e}-n M_{f}$ and $n M_{c}-n M_{f}$.

\section{1 The Reduced-AOG tree}

In the process of calculating effective resistance, we consider $v_{f}^{*}$ one by one. Beginning from $v_{f}^{*}$ and visiting its all ancestors until meeting the root, the visited nodes form an ascendant path, called backbone path. For example, $v_{1}^{*} \rightarrow v_{2}^{*} \rightarrow v_{3}^{*} \rightarrow v_{1}^{+} \rightarrow v_{2}^{+} \rightarrow v_{4}^{*} \rightarrow v_{5}^{*} \rightarrow v_{6}^{*} \rightarrow v_{3}^{+} \rightarrow v_{4}^{+}$is the backbone path in Fig. 4(a). It can be observed that the size of gathering group of $v^{*}$ or $v^{+}$is arbitrary, and $v^{*}$ and $v^{+}$groups are ordered by interlacing manner in the backbone path. In addition, a $v^{*}$-group combining with a $v^{+}$-group forms another kind of group naturally, called $A O G$ (AND-OR-Group), because $v^{*}$ cannot merge with $v^{+}$. AOGs will exist repeatedly in the tree, and thus an AOG can be treated as a basic unit to be processed while reducing the $\mathrm{PN}$ tree.

Definition 1: An $A O G$ in the PN tree has the form like $v_{i}^{*} \rightarrow \cdots \rightarrow v_{n}^{*} \rightarrow v_{j}^{+} \rightarrow \cdots \rightarrow v_{m}^{+}$in the ascendant order. If $v_{t}^{*}=$ $v_{f}^{*}$, it's also called primary-AOG (pAOG); else called extended-AOG (eAOG). Then, the AOG tree is composed of one $p A O G$ and several $e A O G$ s.

Definition 2: A Reduced-primary-AOG (RpAOG) consists of two nodes which are one $v^{*}$ and one $v^{+}$in the sequence of $v_{f}^{*} \rightarrow v^{+}$. A Reduced-extended-AOG $(\operatorname{Re} A O G)$ consists of three internal nodes which are two $v^{*}$ 's and one $v^{+}$in the sequence of $v_{c r}^{*} \rightarrow v_{c l}^{*} \rightarrow v^{+}$or $v_{c l}^{*} \rightarrow v_{c r}^{*} \rightarrow v^{+}$. Note that $v_{c r}^{*}$ and $v_{c l}^{*}$ are the nodes that are visited through their right and left child, respectively. Then, the Reduced-AOG tree is composed of one RpAOG and several ReAOGs.

Rule 2 : Sequential AND nodes in the MTB tree can be always reduced to one- $v^{*}-$ subtree or two- $v^{*}-$ subtree, according to the nodes in the $p A O G$ or in the $e A O G$. From some focused node, say $v_{j}^{*}$, to visit its all ancestors, the left (right) $R_{\text {eff }}$ 's of subtrees of $v_{c r}^{*}$ 's ( $v_{c l}^{*}$ 's) and $v_{j}^{*}$ should be summed up, and denoted by $R_{u}\left(R_{d}\right)$. After all, if all $v^{*}$ 's are in the $p A O G$, they will be merged into one $v^{*}$. The rule can be summarized by the Eqs. 5 and 6 as follows.

$R_{u}=\sum_{i} r_{t_{i}}\left(v_{i}^{*}\right) \cdot \hat{f}\left(v_{i}^{*}\right), i=j, j+1, \ldots, \max$ number of $v^{*}$ in $A O G$.

$R_{d}=\sum_{i} r_{t_{r}}\left(v_{i}^{*}\right) \cdot f\left(v_{i}^{*}\right), i=j, j+1, \ldots, \max$ number of $v^{*}$ in $A O G$

where $f\left(v_{i}^{*}\right)=\left\{\begin{array}{ll}0, & \text { if } v_{i}^{*}=v_{c r}^{*} \\ 1, & \text { if } v_{i}^{*}=v_{c l}^{*}\end{array}\right.$, and $r_{t_{i}}\left(v_{i}^{*}\right)$ and $r_{t_{r}}\left(v_{i}^{*}\right)$ are $R_{\text {eff' }}$ 's of the left and the right subtree of $v_{i}^{*}$, respectively

Rule 3: Sequential OR nodes in the MTB tree can be always reduced to an one- $v^{+}$-subtree. From the lowest node, say $v_{j}^{+}$, to visit its all ancestors, the left (right) $R_{\text {eff }}$ 's of subtrees of $v_{c r}^{+}\left(v_{c t}^{+}\right)$and $v_{j}^{+}$should be summed up, and denoted by $R_{r}\left(R_{l}\right)$. Note that $v_{c r}^{+}$is the node that is visited through its right child. After all, all $v^{+}$'s will be merged into one $v^{+}$. The rule can be summarized by the Eq. 7 as below.

$R_{p}=R_{f} \| R_{r}=\frac{1}{\sum_{i} \frac{1}{r_{t}\left(v_{i}^{+}\right)}}, i=j, j+1, \ldots$, max number of $v^{+}$in $A O G$. (7) where $r_{t}\left(v_{i}^{+}\right)$is the $R_{\text {eff }}$ of the subtree of $v_{i}^{+}$.

According to Rule 2 and Rule 3 , a Reduced-AOG tree can be reduced from $A O G$ tree when all subtrees in the backbone path are replaced with their $R_{e f f}$ 's An example to show the $A O G$ tree is drawn in Fig. 4(a), the Reduced-AOG tree is presented in Fig. 4(b).

\section{2The Reduced-AOG circuit}

The Reduced-AOG circuit is an equivalent topology circuit of the Reduced-AOG tree. The corresponding
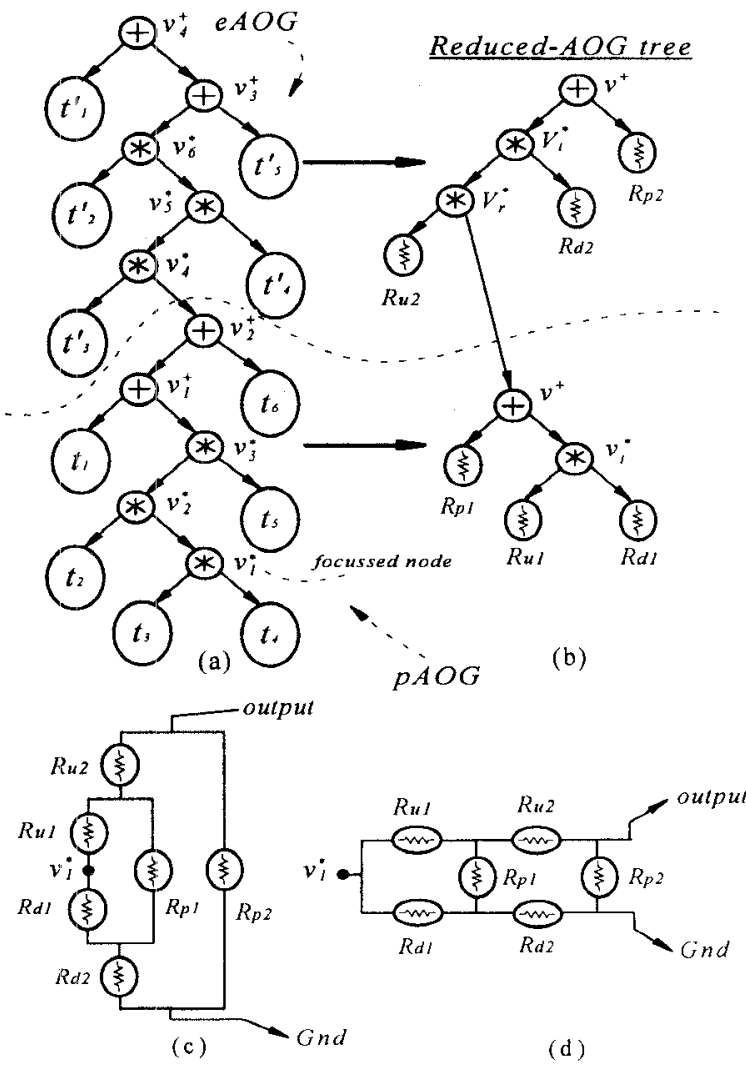

Figure 4: An example for illustrating the reduction of tree. (a) the original PN tree. (b) the Reduced-AOG tree. (c) the Reduecd-AOG circuit. (d) the R-mesh circuit. 
Reduced-AOG circuit of Fig. 4(b) as shown in Fig. 4(c) is a topology connection that is expanded with regard to $v_{i}^{*}$. For each $R_{u i}, R_{d i}$ and $R_{p i}$, it could be open-circuit, shortcircuit or a finite resistance, and these $R_{e f f}$ 's form a block. By arranging the components in the Reduced-AOG circuit, we can find that the Reduced-AOG circuit is exactly an $R$ mesh circuit. Therefore, translating the tree structure (Reduced-AOG tree) to the linked list using AOG as a basic unit (R-mesh circuit) can make the computation of $R_{\text {eff }}$ easier. After translating the Reduced-AOG circuit to the $R$-mesh circuit, we can apply the $Y \rightarrow \Delta$ transformation to the R-mesh circuit and can calculate $R_{\text {eff }}$ block by block. Algorithm EER: (Estimate Effective Resistance)

An $A O G$ tree (also a PN tree) can be reduced to a Reduced-AOG tree by the following steps.

- Step 1: Traverse the tree recursively by in-order manner to select the internal node with charge; of course, leaf nodes and $v^{+}$'s are excluded. Starting from the $v_{f}^{*}$, we can use Rule2 and Rule 3 to reduce the $p A O G$ to the $\operatorname{RpAOG.}$

- Step 2: When visiting to an $e A O G$, also use Rule2 and Rule 3 to reduce the $e A O G$ to the ReAOG. We seek out the first $v_{c r}^{*}$ and $v_{c l}^{*}$ we meet, denoted by $V_{c r}^{*}$ and $V_{c}^{*}$ respectively, and then (1) merge all $v_{c r}^{*}$ into $V_{c}^{*}$ and all $v_{c l}^{*}$ into $V_{c l}^{*},(2)$ add all $R_{\text {eff }}$ 's of the left subtrees of $v_{c r}^{*}$ 's to $R_{e f f}$ of the left subtree of $V_{c}^{*}$, and then can obtain $R_{\mathcal{u}}$. The same action is also applied to the node $V_{c i}^{*}$ to obtain $R_{d i}$. (3) sum up all $R_{e f f}$ 's of $v^{+}$'s to obtain $R_{p i}$.

- Step 3: Repeating step2 until meet the root node, we establish a Reduced-AOG tree.

- Step 4: Translate the Reduced-AOG tree to the corresponding $R$-mesh circuit, and then apply the $Y \rightarrow \Delta$ transformation to the R-mesh circuit. Therefore, we can calculate $R_{e f f}$ block by block.

- Step 5: Calculate the delay value. Then find the next $v_{f}^{*}$ and repeat the above steps again. The algorithm is terminated while the whole tree is completely traversed. \#

To demonstrate Algorithm EER, we use the PN tree that is composed of two $A O G$ s as shown in Fig. 4(a), and its Reduced-AOG tree is shown in Fig. 4(b). Next, the Reduced-AOG circuit and its translated $R$-mesh circuit are shown in Fig. 4(c) and (d), respectively.

\section{Simulation results and conclusion}

This algorithm has been implemented in BTS. The simulation results of 5-input NAND gate with respect to different amount of internal charges are shown in Fig. 5. Another simulation results of the circuit as sketched in Fig. 2(a) are shown in Fig. 6, and there is an about $70 \%$ improvement of the delay time. The complexity of the algorithms grow linearly $(O(n))$ with the size of the cluster, or precisely, with the amount of the internal nodes. The CPU time comparisons are summarized in Table 1, and the speed ratio shows the advantage of BTS, especially for a large-scale circuit. To obtain the more accurate estimation, futher study on the relationship among the delay, the distribution and amount of the internal charges is necessary. This is the future work on BTS development.

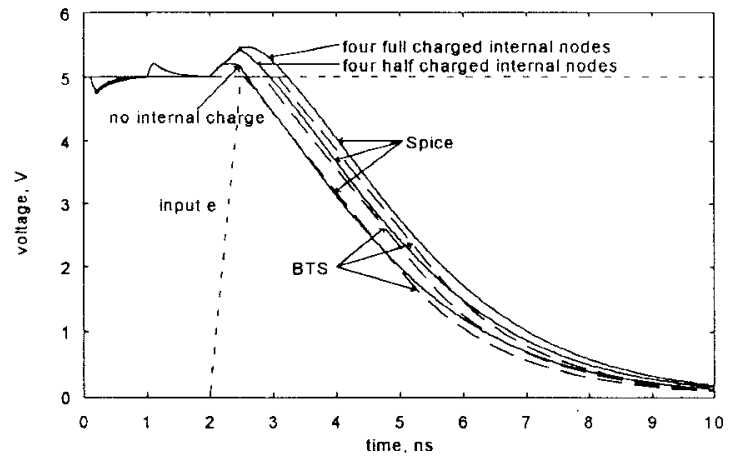

Figure 5: The simulated waveforms of 5-input NAND gate

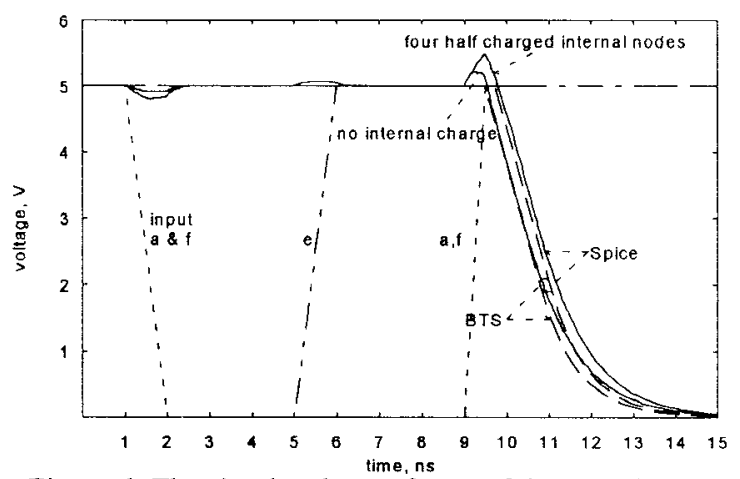

Figure 6: The simulated waveforms of the complex gate of Fig.2(a).

Table 1 : Comparisons between BTS and Spice

\begin{tabular}{|c|c|c|c|c|c|}
\hline Circuit & $\begin{array}{l}\text { MOS } \\
\text { no. }\end{array}$ & $\begin{array}{l}\mathrm{CPU} \\
\text { (DX4 } \\
\text { BTS }\end{array}$ & $\begin{array}{l}\text { on PC } \\
\text {, secs } \\
\text { Pspice }\end{array}$ & Speed ratio & $\begin{array}{l}\text { Primary } \\
\text { input } \\
\text { event no. }\end{array}$ \\
\hline $\begin{array}{l}\text { complex gate } \\
\text { (Fig. 1(a)) }\end{array}$ & 12 & 0.11 & 2.53 & 0.043 & 2 \\
\hline $\begin{array}{l}\text { inverter chain } \\
\text { (100 stages) }\end{array}$ & 200 & 0.28 & 241.18 & 0.0012 & 1 \\
\hline 74138 & 88 & 0.33 & 102.22 & 0.0032 & 11 \\
\hline 7483 & 258 & 0.99 & 774.64 & 0.0013 & 13 \\
\hline 74381 & 584 & 1.10 & 1670.98 & 0.00066 & 14 \\
\hline
\end{tabular}

\section{References}

[1] R. E. Bryant, "A Switch level model and simulator for MOS digital systems," IEEE Computers, vol. C-33, pp. 160-177, Feb. 1894.

[2] C. J. Terman, "RSIM " A Logic-Level Timing Simulator," Proceedings of the IEEE International Conference on Computer Design, New York, pp. 437-440, November 1983.

[3] J. H. Wang, Molin Chang, and W. S. Feng, "Binary-tree timing simulation with consideration of internal charges," IEE Proceedings$\mathrm{E}$, vol. 140, No.4, pp.211-219, July 1993 .

[4] J. Rubinstein, P. Penfield, and M. A. Horowitz, "Signal delay in RC tree networks," IEEE Trans. on Computer-Aided Design, vol. CADtree networks, 3 , pp.202-211, July 1983 .

[5] T. M. Lin, and C. A. Mead, "Signal delay in general RC networks," IEEE Trans. on Computer-Áided Design, vol. CAD-3, No 4, pp. 331 349, October 1984.

[6] J. -P. Caisso, E. Cerny, and N. C. Rumin, "A recursive technique for computing delays in series-parallel MOS transistor circuits," IEEE Trans. on Computer-Aided Design, vol. 10, No.5, pp.589-595, May 1991

[7] F.C.Chang, C.F.Chen, and P.Subramaniam, "An accurate and efficient gate level delay calculator for MOS circuits," Proceedings of 25 th ACM/IEEE conference on Design automation, Anaheim, CA, USA, pp.282-287, June 1988.

[8] T.Horowitz, and S.Sahni, "Fundamentals of Data Structures in Pascal". Computer Science Press, 2nd Edition, Chapter 5, pp.203$271,1987$. 